\title{
Contextualizing Statics: Our Process and Examples
}

\section{Dr. Diana A. Chen, University of San Diego}

Dr. Diana A. Chen is an Assistant Professor of General Engineering at the University of San Diego. She joined the Shiley-Marcos School of Engineering in 2016. Her research interests are in areas of sustainable design, including biomimicry and adaptability in structural, city, and regional applications. She earned her MS and PhD in Civil Engineering from Clemson University in South Carolina, and her BS in Engineering from Harvey Mudd College.

\section{Prof. Sarah Wodin-Schwartz, Worcester Polytechnic Institute}

Dr. Wodin-Schwartz joined WPI in August 2015. She is passionate about teaching core engineering and critical thinking skills that apply to application-driven problem-solving. She is especially interested in engineering design and product development. She is excited to work with students to help them understand not only the technical skills required of them as engineers but also the social, environmental, and physical implications of implementing technical engineering solutions. 


\section{Contextualizing Statics: Our process and examples (WIP)}

Engineering sciences, commonly found in the second year of engineering programs, are the technical courses that are often considered the most foundational for engineering students. Yet, these courses have become impenetrable from changes in content and pedagogy and are often abstracted from any human or societal context. One reason for this abstraction may be due to the understandably prolific use of the "engineering problem-solving method" (EPS) or "engineering as problem definition and solution" (PDS), which teaches students how to methodically approach a technical problem and produce easily checked solutions [1]. However, the EPS method has perpetuated the dominance of mathematical problem solving as the highest value in engineering [2], often unintentionally reinforcing the worldview that technical rigor is more important than the context in which engineering is practiced [1]. This realization has led to a growing body of work documenting efforts towards the contextualization of engineering education [3], [4].

Much of the literature tying engineering education to human or societal context stems from the field of Science, Technology, and Society (STS) and Engineering Studies. Fundamental to STS is the tenant that science and technology are not objective and isolated; rather the creation of new knowledge and technology are influenced by society, politics, and culture, and these technologies in turn will have impacts on society [5]. Over time, this area of work has led to research that examines how integrating socio-technical thinking in the engineering classroom can develop more holistic engineers capable of working in both the social and technical dimensions of contextualized, open-ended problems [6]. Wisnioski, a historian of engineering studies, reflected in his 2015 editorial that, while calls for engineering education reform towards "global, economic, environmental, and societal context" [7] have been existent since as early as the 1920 s, "each wave of reform has come with incomplete efforts to establish institutional rewards that value such historical and contextual work." [8] However, since Wisnioski's editorial, much progress towards contextualization has been made in the engineering community, largely in part due to support from the Accreditation Board for Engineering and Technology (ABET) and the National Science Foundation (NSF), which are two of the largest governing institutions of engineering accreditation and funding, respectively. In the latest revision of student outcomes for accreditation (2019-2020), ABET changed Outcome F from "an understanding of professional and ethical responsibility" to Outcome 4, which specifically adds on that these responsibilities must "consider the impact of engineering solutions in global, economic, environmental, and societal context." [9]. This change in accreditation criteria has been a strong motivator for all engineering programs to include broader contextualization in their curricula. Furthermore, NSF's program for Revolutionizing Engineering Departments (IUSE/Professional Formation of Engineers: RED) has created an avenue through which engineering educators can propose innovative ways to radically change the culture of engineering programs, design revolutionary approaches to engineering and computer science education, and rethink what it means to provide an engineering program [10]. 
Even with this widespread culture change happening in engineering education, implementation at the program and classroom level to align with these new initiatives can be challenging. Below we describe our efforts in bringing context into the Statics classroom through multiple tiers of implementation across two teaching institutions. By contextualizing traditionally abstracted concepts, our goal is to help students understand the role of Statics in engineered objects, as well as enhance student engagement in the material by connecting it to real-world scenarios that they see around them. First, we describe how our institutional contexts have allowed us to pursue this work effectively and with limited risk. Then, we provide examples that we have developed for use in class. Our work builds directly upon Moseley's 2017 paper [1], calling for incremental steps as small as "one paragraph and a few simple questions" with the objective of contextualizing statics. We hope by providing these modularized examples at different degrees of implementability, we can bridge the gap between institutions such as ours, where we can freely experiment with curricula, and others where curricula provides little room for experimentation but may allow for adoption.

\section{Institutional Contexts}

First and foremost, we acknowledge that the work described in this paper is largely thanks to institutional support. At the University of San Diego (USD), where author DAC works, the push towards contextualization in engineering is situated in a larger university mission and strategic vision, a liberal arts University Core curriculum, and an NSF RED grant shared amongst leadership in the School of Engineering, as well as a new engineering department and program committed to helping students understand how their work impacts society. (Details can be found in [11].) Due to this multi-layer united initiative, the new Integrated Engineering program at USD is committed to broadening the definition of engineering and providing students with an engineering education that is explicitly socio-technical in nature. As such, in addition to the work presented here about contextualizing Statics, other ongoing engineering work at USD includes introducing social relevance to heat transfer [12], reimagining energy as an interdisciplinary and decolonized concept [13], integrating social justice into engineering curricula [14], and teaching social responsibility in circuits and materials science [15], [16], among others [17].

At Worcester Polytechnic Institute (WPI), where author SWS works, giving students the opportunity to tackle real world problems began nearly 50 years ago when the curriculum was revolutionized to focus on project based learning (PBL) [18]. This curriculum shift dramatically changed the overall student experience at WPI. Now, with support from the KEEN network (run by the Kern Family Foundation), in addition to the Morgan Center for Teaching and Learning and the Center of Project Based Learning at WPI, bringing contextualized PBL into core engineering classes has taken a more central focus. WPI is committed to forming engineers who are not just technically prepared to perform at a high level, but who are motivated and inspired by the changes they can make in the world around them. 
While the efforts and experimental nature of this work may not be easily replicated from the ground up at other institutions, our hope is that by sharing our class examples, others can build upon our methods or even adopt our materials directly. As we continue to modularize our examples and projects, our goal is to share our work such that contextualization can be readily infused into traditional classrooms without the curricular risk that comes with course development.

\section{Tiers of Statics Context Implementation}

Through discussion of similarities between the two authors' efforts at bringing context to Statics, we discovered that the examples, activities, and projects we developed can be divided into four main categories based on student difficulty level and ease of implementation for the instructor (Table 1). The degree of ease of implementation is a guide based on the authors' experiences of how much preparation time was required to launch the example or assignment in class, how impactful the module was, and how challenging it may be to develop a new one from scratch. Below, we describe and provide examples of each example/assignment type for the reader to adopt as desired.

Table 1: Levels of Statics contextualization type ranked by student difficulty level

\begin{tabular}{|l|l|}
\hline $\begin{array}{l}\text { Contextualization Type } \\
\text { (Ranked by Difficulty Level for Students) }\end{array}$ & Ease of Implementation for Instructor \\
\hline $\begin{array}{l}\text { Level 1: Concept in Context } \\
\text { Demonstrate a Statics concept using real-world } \\
\text { phenomena }\end{array}$ & Least prep time, medium impact, easy effort \\
\hline $\begin{array}{l}\text { Level 2: Problem-Solving in Context } \\
\text { Practice Statics problem-solving in a technical } \\
\text { problem }\end{array}$ & $\begin{array}{l}\text { Medium prep time, high impact, medium } \\
\text { effort }\end{array}$ \\
\hline $\begin{array}{l}\text { Level 3: Decision-Making in Context } \\
\text { Evaluate social and technical decisions through } \\
\text { a class project }\end{array}$ & $\begin{array}{l}\text { Most prep time, requires allocating class } \\
\text { time to introduction and debrief, can have } \\
\text { deep impact if done well }\end{array}$ \\
\hline $\begin{array}{l}\text { Level 4: Make-Your-Own Context } \\
\text { Create new context problems or projects } \\
\text { through a "make-your-own" assignment }\end{array}$ & $\begin{array}{l}\text { Little prep time but needs scaffolding } \\
\text { throughout semester, challenging for } \\
\text { students, high impact if scaffolded well }\end{array}$ \\
\hline
\end{tabular}

\section{Level 1: Concept in Context}

In the simplest way, context can be introduced by explaining new concepts through relatable experiences that students have probably observed before. For example, in San Diego, students likely have tried or observed stand-up paddleboarding during their pastimes on the beach or bay. As a first introduction to moments $(\boldsymbol{M}=\boldsymbol{r} \times \boldsymbol{F})$, two photos of paddleboarding rowing strokes are shown in lecture (see Figure 1). Based on their experience and intuition of 
this physical movement, students are asked to consider which of the two strokes requires a larger moment.
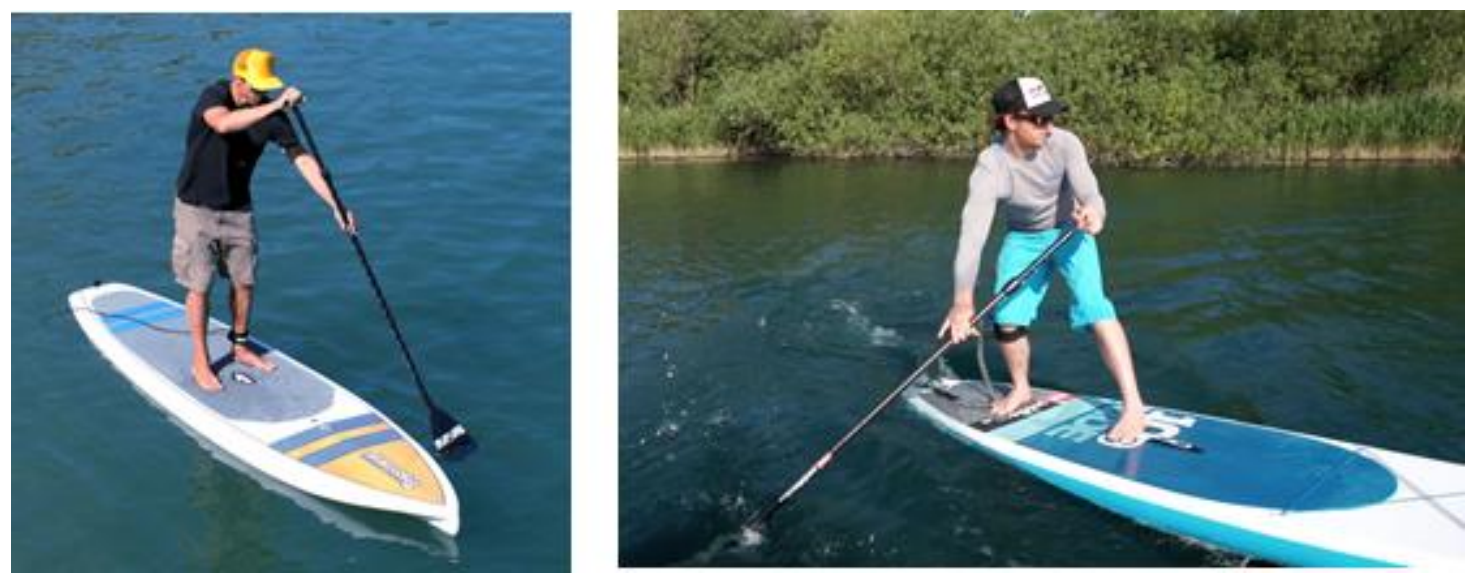

As a first-time paddle-boarder, you are taught two strokes. The first stroke, shown on the left, is kept close to your board. The second stroke, on the right, is very similar, but the paddle is further out from your board.

Which of these strokes is used to paddle forward, and which is used to turn? How do you know?

Figure 1: Level 1 Contextualization Example - Paddleboarding context used to explain moments

This level of contextualization is the easiest to implement in the classroom, as real-world examples can be introduced even on a whim during lecture. Using real-world phenomena to demonstrate unintuitive concepts is particularly effective, since students are able to tie new engineering concepts to what they already know. Other simple examples of this include introducing moments by describing the leverage needed to change a tire, or introducing friction by discussing the differences of driving on a dry road and hydroplaning on an icy road. As long as the instructor is proficient at identifying Statics in the world around them, they can help students begin to draw these connections themselves.

\section{Level 2: Problem-Solving in Context}

The second level of contextualization, where entire technical problems are framed within a context, is best implemented as in-class problem-solving practice with an opportunity for debriefing but can also be assigned for homework. These types of problems are well-defined with an introduction and purpose to solving, and ask the students to reflect on the value of their solution. For example, in his 2017 paper calling for context in Statics, Moseley poses a friction problem in which the mathematical solution emphasizes the need for fixing bookcases to the wall, lest they are tipped over by - and on to - toddlers [1]. Sometimes the mathematical solution provides insight on why certain products are designed how they are, or compels the student to 
consider alternative reasons why products are not designed according to "the best solution." In Figure 2, we share a practice problem that considers a backpacking pack as a composite shape where students solve for the center of gravity and reflect on why backpackers are not advised to pack their packs in a more weight-efficient way.

\section{Context Example: Packing a Backpacking Pack}

\section{https://www.rei.com/learn/expert-advice/loading-backpack.html (video)}

When backpacking, it is important to pack your backpack correctly to maximize your hiking stamina over long distances and high elevation gains, and minimize the effort your body puts into carrying an inefficient load. Experts claim that the weight distribution in the given image is best.

Where is the center of gravity for this pack? The backpack has dimensions 25 "x 13 "x 8 ". Assume $\mathrm{A}=6 \mathrm{lbs}, \mathrm{B}=10 \mathrm{lbs}, \mathrm{C}=3 \mathrm{lbs}, \mathrm{D}=5 \mathrm{lbs}$, and neglect the weight of the backpack itself. Make other assumptions as needed.
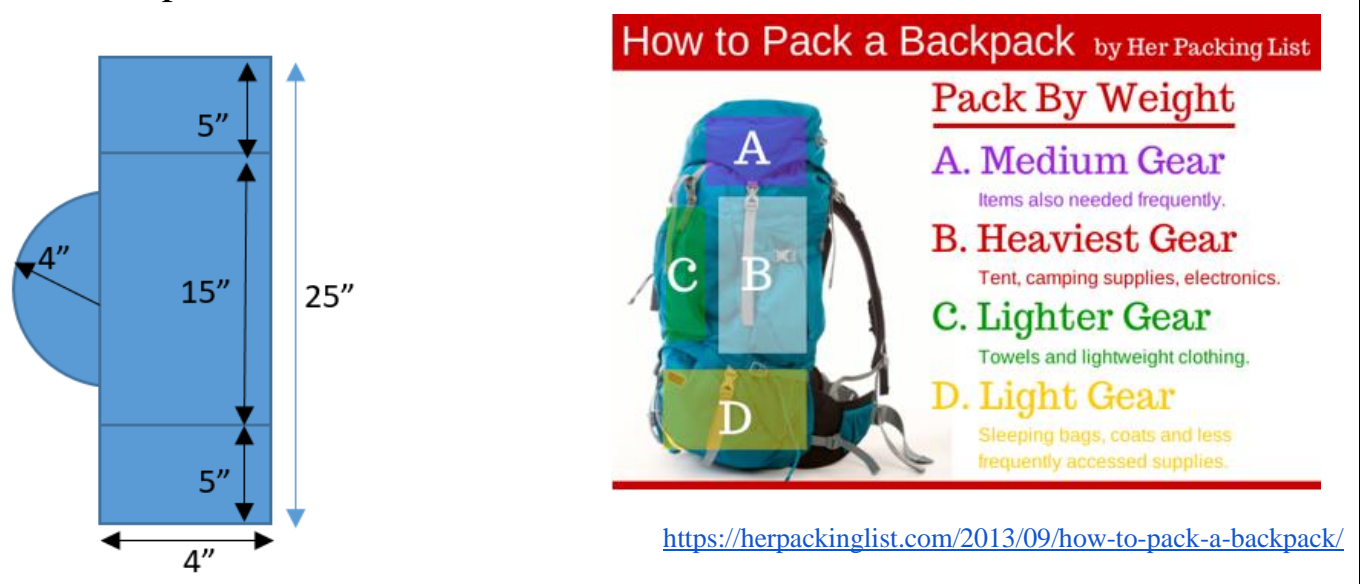

Reflect:

1. The backpack is held up by your shoulders and hips. Which of these should support the more weight to maximize efficiency?

2. The video says to tighten the compression straps once the pack is on to stabilize and streamline the pack. Where might these straps be placed, and how should they shift the load of the pack?

3. Does the expert way to pack the backpack align with how you would pack it given what you know about center of gravity? Why do you think this is?

Figure 2: Level 2 Contextualization Example - Backpacking context problem used to explain composites and center of gravity

This level of contextualization requires more preparation and creativity to implement. In order to create an entire problem in context, the instructor needs to be aware of Statics in their own surroundings in order to first, draw the connection, and second, formulate the parameters of 
the problem. In addition to creating a problem statement that pushes students to consider the meaning of the numerical solution in the original context, the inclusion of reflection questions helps to emphasize how oftentimes a situation may be influenced by more than just the technical parameters provided.

\section{Level 3: Decision-Making in Context}

The third level of contextualization is to create a class project that requires students to utilize engineering decision-making to evaluate different potential solutions to a given context problem. One project that has been developed by author SWS and widely shared through active learning and problem-based learning networks [19], [20], named Flying Forces, asks students to consider the sociotechnical nature of engineering decision-making.

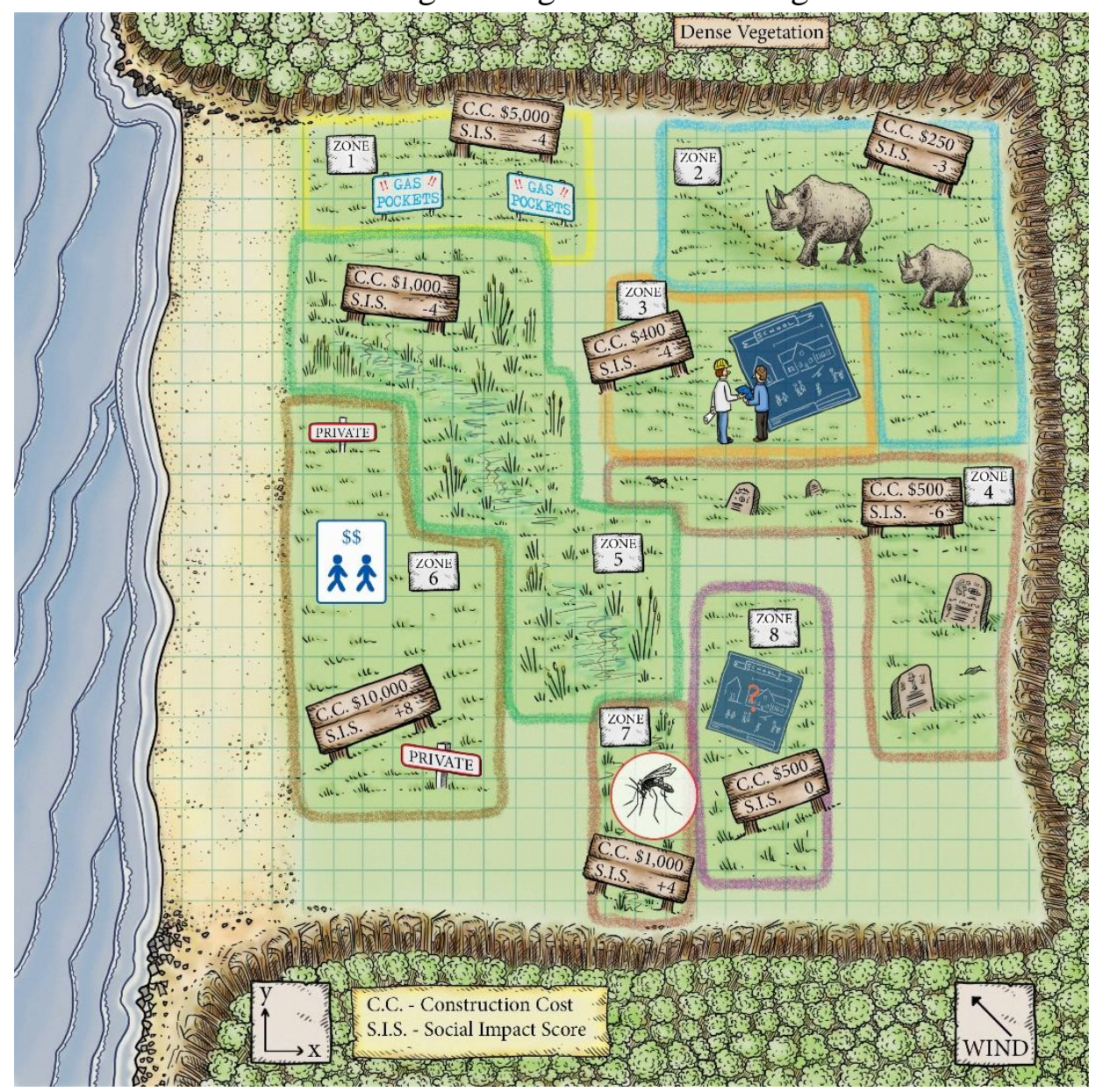

Figure 3: Fictional map used in Flying Forces project [19]

In the Flying Forces project, students work in teams to develop an anchoring system for a floating wind turbine to bring context to the technical skills associated with analyzing 3D particle equilibrium. While this concept can be demonstrated with a theoretical point floating in space, developing a project around the concept gives student the opportunity to personally connect with the technical work. For this project, students are given a map of a fictional village divided into eight zones in which wind turbine anchors can be placed (see Figure 3). Construction costs and 
social impacts must be taken into consideration and are based on the scenario described within each zone. Negative social impacts include environmental effects, such as $\mathrm{CO} 2$ emissions from developing swamp lands, the potential disturbance of religious burial grounds, and disturbing animal migration routes. Positive impacts include the reduction of mosquitos and job creation. In addition to considering the social ramifications of construction based on their selected zones, students research a location of their choice and propose technologies for use in that community. They evaluate the social impact of these technologies and incorporate a qualitative analysis into an overall social impact report. This project gives students the chance to solve a technical challenge that is inextricably linked to social factors within the community. By integrating projects into an earlier course of the engineering curriculum, students learn early on that engineering requires a broad range of skills, is not always an exact, straightforward process, and that there is rarely ever one "right" answer.

Author SWS has implemented this project 3 times over the course of 3 years and is still making minor improvements to the assignment. Author DAC implemented a scaled-down version of this project for the first time in Fall 2018 with approximately 70 students and plans to continue iterating on a scaled-down version of the assignment. For the interested reader, details of assigning this project (including lesson plans, handouts, and instructors notes) can be found at www.engineeringunleashed.com with the creation of a free account.

\section{Level 4: Make-Your-Own Context}

While simply creating one homework problem may seem simple, asking students to make their own context problems is the most challenging task for them within the four levels of contextualization. (A term project may be time-intensive for students, but creation lies within the top tier of Bloom's Taxonomy.) In the Make-Your-Own Context problems, students are asked to create their own problem statement, draw free body diagrams of the objects under analysis, and estimate the loads applied to that system. To push students even further, a variant on the assignment is to ask students to create a problem that, when solved, would inform human behavior or decision-making through its solution and associated reflection questions.

To start, students are encouraged to consider their own hobbies or extracurricular activities for inspiration. This may lead them to wanting to conduct an analysis on a piece of sport equipment or ask a question regarding a simplified model of a physical activity that involves balanced forces. For example, past students have created problems about workout efficiency due to various angles of pushing or pulling. One student created a problem modeling a heavy tray carried by a waitress - this simplified biomechanics model examined the various angles of the weight and analyzed whether the tray would be less tiring to carry with an extended arm or by placing the hand near the body.

A specific criterion to this type of contextualization is that the focus should be on answering a question through the statics analysis rather than formulating a question around an existing mechanical product. From experience, students who fixate on the product rather than its functions often produce poor analyses. For example, one student created a Statics problem 
around rock climbing with a cam. While the cam is a mechanical device that could have easily been analyzed with simple forces, the student instead investigated the angle at which he should insert the cam into a crack to best support his weight on an icy day. By not only writing but also solving the problem, he was able to reinforce his rock climbing heuristics with his own proof of calculations.

While this project is only a 1-page submission with an introduction, problem, and reflection questions, students spend considerable time formulating viable ideas and technical parameters for the problem, often in consultation with the instructor. Author DAC has implemented the "make-your-own" problem as a project two years in a row, once as extra credit and once as a required project. Both iterations have offered extra credit for submitting an additional technical report that documents the context and provides a written analysis and solution. To raise the stakes for the project, students have been required to submit their work to www.engineeringincontext.com, a website created and managed by Dr. Sean Moseley at RoseHulman Institute of Technology. Engineering in Context is an open-source database for Statics context problems for Statics instructors to draw from.

\section{Author Tips and Reflections}

Envisioning, let alone creating, these connections between context and Statics concepts can be difficult. In our experiences of sharing our work, we are often asked how we go about coming up with these examples and how to identify relevant contexts. Here we offer our reflections on the process and tips on how to conceptualize context around Statics to help interested instructors generate their own ideas.

- Think about Statics in your activities and daily routine, and write a note when you think of a Statics connection.

Statics is everywhere around us. Keep your Statics lesson planning in mind particularly when doing activities you enjoy and find interesting, since students will pick up on your energy when you share your example. For instance, the backpack example shared above was inspired by an actual backpacking trip where DAC was fiddling with compression straps, trying to get the backpack to sit correctly. Bringing the backpack to class for this practice problem also helped students engage and wrap their head around the weight distribution that they could physically see. However, your daily routine can also inspire Statics problems. For example, on a trip through the grocery store, SWS discovered that a significant couple moment was required to turn the shopping cart with one hand into the next aisle. No carts were stolen for a class demonstration, but rolling chairs in the classroom easily replicated the context for students to experience ways in which they see couple moments in their daily lives.

- Provide opportunities for students to draw their own connections.

Once you start noticing Statics all around you, you will see how much deeper your personal connection to Statics becomes. Providing students opportunities to make these 
connections will be just as, if not more, powerful for them. Having students think about their passions like backpacking, sports, theater, art, or food, etc. and how these connect back to the course content will enrich their experience. Give students space to reflect on the solutions for and discussions about context problems. It's in these spaces that students are able to vocalize why the answer is significant to them. For example, after finishing the backpacking example in class, one student commented that his ROTC unit planned to do a 70-lb ruck march the next day and that the example in context inspired him to think about how to pack his bag efficiently. Also, after solving Moseley's friction example in the context of a bookshelf being tipped over by a toddler, one student with a toddler shared with the class how the example hit home for him, and how he would mount his furniture that weekend.

Our goal isn't only to have students make their own technical problems, but to help students start seeing their world through a technical engineering lens, such as being able to identify load paths and moments all around them. This type of learning can be very challenging for students. Many students have never had the opportunity to make these personal connections, so it may push them out of their comfort zone. Be upfront with your students. Let them know that the process of developing their own connections is difficult. It is likely that there is no single, correct answer. Some students find it helpful to sit down and talk about their ideas with peers, TAs or instructors.

- Be ready to go back to first principles, and make model assumptions as needed.

As you work through context problems with students, it's likely that you may run into instances of confusion yourself since simplifying real-world scenarios into static and determinant problems can require many assumptions. Be okay with responding with "I don't know" to be transparent with students about how real context, while important, can be challenging. It's also helpful to work through a problem with the students ("Let's think about this") for them to see your thinking process in drawing connections between course content and real analyses. Often multiple assumptions will need to be made to ensure a problem can be solved at an appropriate difficulty level. For example, in a biomechanics problem, you may want to model muscles as springs, bones as bars, or the wrist as a fixed connection between the hand and arm, depending on the problem scope.

- Covering context of one problem can be more impactful in the long run than covering multiple problems devoid of context.

One of the challenges of teaching lower level fundamental courses is the pressure to cover all the content in the textbook to ensure students can succeed in follow-on courses. This pressure can lead to a math- and physics-heavy course, but succumbing to this default results in providing students few opportunities to apply their skills. Students may leave the course capable of performing solving processes by rote memory, but they may very well lack the skills required to identify that the problem is, in fact, a Statics analysis. This gap in understanding isolates the fundamental skills learned in different engineering courses, making it difficult for students to 
later apply the skills they have learned when outside of that course's homework. By giving students fewer but more contextualized problems in the early core courses, students will be able to identify what type of analyses are necessary in their later courses and project work.

Additionally, adding context gives students more opportunities to personally connect with the content, which can lead to greater interests and engagement with the topic. Between the two authors, there have been many times when students have returned after a class session or even after the semester to comment on a project or contextualized example they remember from the course, but there is rarely, if ever, a student who returns to chat about one of the many strange pipe configurations or masses hanging from suspended cables.

\section{Conclusion}

As engineering professors, we are forming the engineers of tomorrow. If we want our students to tackle the grand challenges of our time, we need to train them to apply their skills to those challenges. These challenges are not simple, clean, and tidy like a textbook problem with an answer in the back of the book. Defining and analyzing problems is far more difficult than solving simplified textbook problems, and the engineers of tomorrow need to be able to draw the connections between their technical skills and how they will be applied in the real world.

By adding context to Statics problems, our goal is to help students see very early on that technical problems are rarely solved in isolation, as engineering is a profession grounded in an often messy reality. Using different levels of contextualization can scaffold the student experience, giving them the confidence to try new things and make personal connections to the engineering field. In this paper, we described four levels of contextualization that we implement in the Statics classroom, ranging from low-stakes conceptual examples to class projects. Using real-world experiences to demonstrate new Statics concepts is a great way to get started. Projects are another a great way to sustain student engagement and can give them a wider perspective on potential impacts of engineering decision-making. Finally, challenging students to develop their own problems pushes them to make lasting connections to the material.

Adding context to Statics can be a challenge because it requires reaching beyond the current expectations of most Statics courses. In addition, the time and effort needed to develop and integrate this new type of course content can be intensive. Our hope is that by presenting some of our own work, methods, and tips, other Statics instructors are able to pave their own path towards contextualizing Statics in their own classrooms. While interested readers are encouraged to adopt the examples and projects we have presented here as a starting point, we hope our work will inspire others to develop their own context examples to, in turn, inspire their own students to see Statics everywhere in the world around us.

\section{References}

[1] S. Moseley, "One Paragraph and a Few Simple Questions - Giving Statics Problems Human Context," in ASEE Annual Conference and Exposition, Conference Proceedings, 2017, p. 6.

[2] G. L. Downey, "PDS: Engineering as Problem Definition and Solution," Springer, Cham, 
2015, pp. 435-455.

[3] D. Riley, "Engineering and Social Justice," Synth. Lect. Eng. Technol. Soc., vol. 3, no. 1, pp. 1-152, Jan. 2008.

[4] J. Lucena, J. Schneider, and J. A. Leydens, "Engineering and Sustainable Community Development," Synth. Lect. Eng. Technol. Soc., vol. 5, no. 1, pp. 1-230, Jan. 2010.

[5] L. Winner, "Do Artifacts Have Politics?," Daedalus, vol. 109, no. 1, pp. 121-136, 1980.

[6] J. A. Leydens, K. Johnson, S. Claussen, J. Blacklock, B. M. Moskal, and O. Cordova, "Measuring Change over Time in Sociotechnical Thinking: A Survey/validation Model for sociotechnical Habits of Mind," in ASEE Annual Conference and Exposition, Conference Proceedings, 2018.

[7] Engineering Accreditation Commision, "CRITERIA FOR ACCREDITING ENGINEERING PROGRAMS Effective for Reviews During the Engineering Accreditation Commission," Baltimore, 2014.

[8] M. Wisnioski, "What's the Use? History and Engineering Education Research," J. Eng. Educ., vol. 104, no. 3, p. n/a-n/a, Jun. 2015.

[9] “Criteria for Accrediting Engineering Programs, 2019 - 2020 | ABET.” [Online]. Available: https://www.abet.org/accreditation/accreditation-criteria/criteria-foraccrediting-engineering-programs-2019-2020/\#GC3. [Accessed: 05-Mar-2019].

[10] "IUSE/Professional Formation of Engineers: Revolutionizing Engineering Departments | NSF - National Science Foundation." [Online]. Available: https://www.nsf.gov/funding/pgm_summ.jsp?pims_id=505105. [Accessed: 02-Feb-2019].

[11] D. A. Chen and G. D. Hoople, "Contextualizing a New General Engineering Curriculum in the Liberal Arts," 2017 ASEE Annu. Conf. Expo., Jun. 2017.

[12] E. A. Reddy, B. Przestrzelski, S. M. Lord, and I. Khalil, "Introducing Social Relevance and Global Context into the Introduction to Heat Transfer Course," in ASEE Annual Conference Proceedings, 2018.

[13] G. D. Hoople, J. A. Mejia, D. A. Chen, and S. M. Lord, "Reimagining Energy: Deconstructing Traditional Engineering Silos Using Culturally Sustaining Pedagogies." 23-Jun-2018.

[14] J. A. Mejia, D. A. Chen, O. O. Dalrymple, and S. M. . Lord, "Revealing the Invisible: Conversations about -Isms and Power Relations in Engineering Courses," in ASEE Annual Conference Proceedings, 2018.

[15] B. Przestrzelski, E. A. Reddy, and S. M. Lord, "Integrating Experiential with Technical: How Materials Science Modules Can Help Redefine the Traditional Engineering Canon," in ASEE Annual Conference Proceedings, 2018.

[16] S. M. Lord, B. Przestrzelski, and E. Reddy, "Teaching Social Responsibility: Conflict Minerals Module for a Circuits Class," in 2018 World Engineering Education Forum Global Engineering Deans Council (WEEF-GEDC), 2018, pp. 1-6.

[17] S. M. Lord et al., "Creative Curricula for Changemaking Engineers," in 2018 World Engineering Education Forum - Global Engineering Deans Council (WEEF-GEDC), 2018, pp. 1-5.

[18] “Center for Project Based Learning » WPI Plan.” [Online]. Available: https://wp.wpi.edu/projectbasedlearning/proven-pedagogy/bernard-m-gordon-award/wpiplan/. [Accessed: 04-Feb-2019].

[19] S. Wodin-Schwarz, "Flying Forces: Adding Lift to Statics," KEENzine 5 - The Handbook for EML, pp. 46-49. 
[20] “Center for Project Based Learning» Workshops \& Seminars." [Online]. Available: https://wp.wpi.edu/projectbasedlearning/workshops-seminars/. [Accessed: 04-Feb-2019]. 